\title{
L5 Spinal Nerve Axotomy Induces Distinct Electrophysiological Changes in Axotomized L5- and Adjacent L4-Dorsal Root Ganglion Neurons in Rats In Vivo
}

\author{
Laiche Djouhri, ${ }^{1}$ Asad Zeidan,, Mohammad Alzoghaibi, ${ }^{2}$ Mohammad F. Al Otaibi, ${ }^{2}$ and Seham A. Abd El-Aleem ${ }^{3,4}$
}

\begin{abstract}
Peripheral neuropathic pain (PNP) is a major health problem for which effective drug treatment is lacking. Its underlying neuronal mechanisms are still illusive, but pre-clinical studies using animal models of PNP including the L5-spinal nerve axotomy (L5-SNA) model, suggest that it is partly caused by excitability changes in dorsal root ganglion (DRG) neurons. L5-SNA results in two DRG neuronal groups: (1) axotomized/damaged neurons in L5- plus some in L4-DRGs, and (2) ipsilateral L4-neurons with intact/uninjured fibers intermingling with degenerating L5-fibers. The axotomized neurons are deprived of peripherally derived trophic factors and degenerate causing neuroinflammation, whereas the uninjured L4-neuorns are subject to increased trophic factors and neuroinflammation associated with Wallerian degeneration of axotomized L5-nerve fibers. Whether these two groups of DRG neurons exhibit similar or distinct electrophysiological changes after L5-SNA remains unresolved. Conflicting evidence for this may result from some studies assuming that all L4-fibers are undamaged. Here, we recorded somatic action potentials (APs) intracellularly from C- and A-fiber L4/L5 DRG neurons in vivo, to examine our hypothesis that L5-SNA would induce distinct electrophysiological changes in the two populations of DRG neurons. Consistent with this hypothesis, we found (7 days post-SNA), in SNA rats with established pain hypersensitivity, slower AP kinetics in axotomized L5-neurons and faster AP kinetics in L4-nociceptive neurons including decreased rise time in $\mathrm{A} \delta$-and $\mathrm{A} \beta$-fiber nociceptors, and after-hyperpolarization duration in $\mathrm{A} \beta$-fiber nociceptors. We also found several changes in axotomized L5-neurons but not in L4-nociceptive neurons, and some changes in L4-nociceptive but not L5-neurons. The faster AP kinetics (decreased refractory period) in L4-nociceptive neurons that are consistent with their reported hyperexcitability may lead to repetitive firing and thus provide enhanced afferent input necessary for initiating and/or maintaining PNP development. The changes in axotomized L5-neurons may contribute to the central mechanisms of PNP via enhanced neurotransmitter release in the central nervous system (CNS).
\end{abstract}

Keywords: axotomy; neuropathic pain; nociception; partial nerve injury; primary sensory neurons; uninjured fibers

\section{Introduction}

$\mathbf{C}$ Hronic Peripheral neuropathic Pain (PNP), pain caused by a lesion or disease of the somatosensory system ${ }^{1}$ is a major health problem that affects $\sim 8 \%$ of the general population. ${ }^{2} \mathrm{PNP}$ is resistant to currently available drugs, and is characterized, in humans, by hypersensitivity to normally painful (hyperalgesia) and non-painful (allodynia) stimuli, and spontaneous/ongoing pain. ${ }^{3}$ Pre-clinical studies using animal models of PNP including the L5 spinal nerve ligation/axotomy (L5-SNL/SNA) model $^{4}$ suggest that PNP is caused, at least partly, by abnormal hyperex- citability of dorsal root ganglion (DRG) neurons. ${ }^{5,6}$ The L5-SNL/ SNA model results in two main DRG neuronal groups: (1) neurons axotomized/damaged during surgery, mostly in L5 "L5-axotneurons" plus some in L4 "L4-axot-neurons"; these neurons are deprived of peripherally derived trophic factors and degenerate causing neuroinflammation, and (2) ipsilateral L4-DRG-neurons with intact/uninjured-fibers intermingling with degenerating L5-fibers, which conduct nerve impulses to the central nervous system (CNS) from their receptive fields: "L4-RF-neurons." These L4-neurons are subject to influences of the neuroinflammation associated with Wallerian degeneration of axotomized

\footnotetext{
${ }^{1}$ Department of Basic Medical Sciences, College of Medicine (QU Health), Qatar University, Doha, Qatar.

${ }^{2}$ Department of Physiology, College of Medicine, King Saud University, Riyadh, Saudi Arabia.

${ }^{3}$ Department of Histology and Cell Biology, University of Manchester, Manchester, United Kingdom.

${ }^{4}$ Department of Pathology, Faculty of Medicine, Minia University, Minia, Egypt.
} 
L5-fibers and increased levels of peripherally derived trophic factors because of a lack of uptake by the axotomized neurons. ${ }^{7}$ These opposing influences suggest that these two neuronal groups (L5-axot-neurons and L4-RF-neurons) should exhibit opposing changes.

A few ex vivo or in vitro studies compared the electrophysiological changes in "L5-axot-neurons" and adjacent ipsilateral L4-neurons following L5-SNL/SNA, ${ }^{8-10}$ and reported either changes in "L5-axot-neurons" only ${ }^{9,10}$ or similar changes in "L5-axot-neurons" and L4-neurons. ${ }^{8}$ However, identification of the receptive properties (receptive fields) of L4-neurons was not possible in the in vitro studies. ${ }^{9,10}$ Even in the ex vivo study by Ma and coworkers, ${ }^{8}$ only a few of the L4-neurons were identified as "L4-RF-neurons." Therefore, whether "L5-axot-neurons" and adjacent "L4-neurons" exhibit similar or distinct changes in their electrophysiological properties after L5-SNL/SNA, remains unresolved. The conflicting evidence for this may result from some studies assuming that all L4-fibers are undamaged. Furthermore, there have been no in vivo studies that compared electrophysiological properties of these two groups of DRG neurons after L5-SNL/SNA. Even in our previous in vivo study ${ }^{6}$ in which we reported some changes in electrophysiological properties of L4DRG neurons after L5-SNA, we did not examine changes in electrophysiological properties of axotomized L5-DRG neurons.

Using immunostaining with activating transcription factor 3 (ATF3) (a neuronal injury marker), we ${ }^{11}$ and others ${ }^{12,13}$ have previously shown that a large percentage of L4-DRG neurons (range from $11 \%$ to $>35 \%$ ) were ATF3-positive following L5 spinal nerve injury. More importantly, surgical exposure of L5 spinal nerve induces ATF3 in the L4-DRG neurons, irrespective of whether the L5 nerve is subsequently cut, ${ }^{13}$ indicating that a substantial proportion of L4-DRG neurons are unintentionally damaged during the L5-SNA surgery. These injured L4 neurons "L4-axot-neurons" are likely to exhibit similar phenotypic changes to those of "L5-axotneurons." To exclude those "L4-axot-neurons," in the present study, we examined electrophysiological changes in "L5-axotneurons" and adjacent physiologically identified conducting L4neuorns; that is, "L4-RF-neurons" 7 days after L5-SNA in vivo. In other words, all our L4-neurons in SNA rats were L4-RF-neurons. As noted, "L5-axot-neurons" are deprived of target-derived trophic factors such as nerve growth factor (NGF), and are therefore referred to as "undertrophed,"7 whereas "L4-RF-neurons" are "overtrophed" by NGF and brain derived neurotrophic factor (BDNF). ${ }^{14,15}$ Consistent with the opposing influences on "L5-axotneurons" and adjacent conducting "L4-RF-neurons," we found, in the present study, opposite changes in these neuronal groups or changes in one group only.

\section{Methods}

\section{Animals and in vivo preparation}

Experiments (sodium pentobarbitone $60 \mathrm{mg} / \mathrm{kg}$ i.p.) were conducted on young adult female Wistar rats (150-180 g) under deep anesthesia. All the experimental procedures (i.e., surgery and recordings) complied throughout with the 1986 UK Scientific Procedures Animals Act. At the end of experiments, animals were killed with an overdose of sodium pentobarbitone. Two groups of rats were used in the present experiments. The first was a normal untreated group of animals that had had no prior surgery; the normal L4 and L5 DRGs are abbreviated in Figures and Table 1 to "L4/5 Norm." We did not use a separate sham-operated group because: (1) sham operation (surgical exposure of the L5 spinal nerve without its subsequent transection) results in a nearly identical number of injured neurons (ATF3-positive) in the L4 DRG as the L5 spinal nerve transaction, ${ }^{13}$ and (2) the mean values of action potential (AP) variables recorded intracellularly in DRG neurons from sham operated rats ${ }^{8,16}$ did not differ from those recorded from unoperated normal rats ${ }^{6,17,18}$ (see also the present study). Therefore, the most appropriate control for comparison with nerve injury is no nerve injury.

The second group was designated the SNA group. The L5-SNA model of PNP is a modified version of the original SNL (Chung) model $^{4}$ and was produced as described previously. ${ }^{6,11}$ Briefly, under sterile conditions, an incision was made at the level of the lumbar spine to expose and remove the left transverse process of the L6 vertebra. The ventral ramus of L5 spinal nerve was then isolated, and tightly ligated with a 6-0 silk suture. To prevent fiber regeneration, the ligated L5 spinal nerve was transected just distal to the suture, with care taken to minimize damage to the L4 spinal nerve. The skin incision was then closed with intracutaneous sutures. Healing occurred in all cases by day 7 after surgery. It is noteworthy that a small population of L5 DRG neurons that project to the L5 dorsal primary ramus may be intact. Therefore, it is likely that there is a small contamination of the presumed axotomized L5 population with intact fibers.

All neurons from the adjacent ipsilateral L4 DRG that are included and referred to in Figures and Table 1 as "L4 SNA" were functionally identified. That is, they all had identified sensory receptive fields and conducted APs centrally from their peripheral targets. They are referred to throughout this article as "L4-RFneurons." For a detailed explanation of the importance of including only L4 neurons with identified sensory receptive fields, see the study by Djouhri and coworkers. ${ }^{6}$

Table 1. Summary of Changes in Electrophysiological Properties of L5-Axot-Neurons AND L4-NOCICEPTORS 7 DAYS AFTER L5-SNA

\begin{tabular}{|c|c|c|c|c|c|c|c|c|c|c|c|}
\hline$C V$ range & Animal group & $\mathrm{n}$ & $C V(\mathrm{~m} / \mathrm{s})$ & $\begin{array}{c}E m \\
(-m V)\end{array}$ & $\begin{array}{c}A P \text { height } \\
\quad(m V)\end{array}$ & $\begin{array}{c}A P O V S \\
(\mathrm{mV})\end{array}$ & $\begin{array}{c}A P D B \\
(m s)\end{array}$ & $\begin{array}{l}\text { Rise time } \\
\quad(m s)\end{array}$ & $\begin{array}{l}\text { Fall time } \\
(\mathrm{ms})\end{array}$ & $\begin{array}{c}A H P \\
A M P(m V)\end{array}$ & $\begin{array}{c}A H P \\
80 \%(m s)\end{array}$ \\
\hline & L5-AXOT & 10 & - & - & $\downarrow \downarrow$ & $\downarrow \downarrow$ & $\uparrow$ & $\uparrow$ & - & - & - \\
\hline \multirow{2}{*}{ C-fiber } & L4 NOCI SNA & 11 & - & - & - & - & - & - & - & $\downarrow$ & - \\
\hline & L5 AXOT & 15 & - & - & - & - & $\uparrow \uparrow \uparrow$ & - & $\uparrow \uparrow \uparrow$ & $\downarrow \downarrow$ & $\downarrow \downarrow \downarrow$ \\
\hline $\mathrm{A} \delta$-fiber & L4 NOCI SNA & 14 & - & - & $\uparrow \uparrow$ & - & - & $\downarrow$ & - & - & - \\
\hline \multirow{2}{*}{$\mathrm{A} \beta$-fiber } & L5 AXOT & 30 & $\downarrow$ & $\uparrow$ & $\downarrow \downarrow$ & $\downarrow$ & $\uparrow \uparrow \uparrow$ & $\uparrow \uparrow \uparrow$ & $\uparrow \uparrow \uparrow$ & $\downarrow \downarrow \downarrow$ & $\uparrow$ \\
\hline & L4 NOCI SNA & 15 & - & - & - & $\uparrow \uparrow$ & - & $\downarrow$ & - & - & $\downarrow$ \\
\hline
\end{tabular}

Mann-Whitney test was used to compare L5 axotomy groups with all normal (for $n$ in all normal group, see figures).

One arrow, $p<0.05$; two arrows, $p<0.01$; three arrows $p<0.001$.

SNA, spinal nerve axotomy; CV, conduction velocity; RMP, resting membrane potential; AP, action potential; OVS, overshoot; DB, duration at base; AMP, amplitude. 


\section{Pain behavioral testing}

We have previously shown that SNA rats exhibit behavioral indices of mechanical and heat hypersensitivity, but not spontaneous/ongoing pain. ${ }^{6,11}$ To confirm that the SNA rats used in the present study also show PNP behaviors, pain behavioral testing was assessed in 10 SNA rats. Briefly, paw withdrawal threshold (PWT), paw withdrawal latency (PWL), and cold escape/nocifensive behavior were assessed on the ipsilateral (left) hind paw of each SNA rat. As reported previously, ${ }^{6,11}$ mechanical and heat hypersensitivities were indicated by a significant decrease in the mean PWT and PWL respectively, whereas cold hypersensitivity was indicated by a significant decrease in the duration of cold-evoked responses. The tests were performed 1 day before induction of SNA to determine the baseline values, and 7 days after SNA to determine whether SNA rats develop PNP behaviors. An average of three separate trials with at least $1 \mathrm{~h}$ separating each trial was used.

\section{Intracellular electrophysiological recordings}

Recordings were made 7 days post-operatively or in unoperated normal rats of similar age/weight. At the time of recording, rats weighed $150-180 \mathrm{~g}$. Full details of the animal preparation were as reported previously in the rat. ${ }^{19,20}$ Briefly, to allow artificial ventilation and monitoring of end-tidal $\mathrm{CO}_{2}$, a tracheotomy was performed. End-tidal $\mathrm{CO}_{2}$ was maintained between $3 \%$ and $4 \%$ by adjusting the rate and volume of the respiratory pump. To enable regular injections of additional doses of anesthetic and to monitor blood pressure, the left jugular vein and carotid artery were cannulated. Any rats with blood pressure $<80-100 \mathrm{~mm} \mathrm{Hg}$ were excluded.

A laminectomy from vertebrae L2 to L6 was performed to expose the L4 and L5 DRGs and their corresponding dorsal roots. The exposed nervous tissue was covered with warmed paraffin oil $\left(30^{\circ} \mathrm{C}\right)$ in a large paraffin pool that was constructed using dental impression material. To improve recording stability, a muscle relaxant (pancuronium, $0.5 \mathrm{mg} / \mathrm{kg}$ ) was administered intravenously to all rats during intracellular recordings. This was repeated approximately every hour and was always accompanied by an additional dose $(10 \mathrm{mg} / \mathrm{kg}$, i.v.) of the anesthetic. The dose and frequency of the additional anesthetic was the same as that required to maintain complete areflexia (absence of limb withdrawal reflexes to a noxious pinch) in the absence of muscle relaxant during the preceding $2-3 \mathrm{~h}$ surgery period. The temperature in the paraffin pool measured near the DRG under study was maintained throughout close to $\sim 30^{\circ}$ (mean $30.8^{\circ} \mathrm{C}$; range $28-32^{\circ} \mathrm{C}$ ).

Sharp glass microelectrodes filled with $3 \mathrm{M}$ or $1 \mathrm{M} \mathrm{KCl}$ were used for intracellular voltage recordings as described previously. ${ }^{19,21}$ Somatic APs were antidromically evoked by electrical stimulation of the L4 or L5 dorsal roots with single rectangular pulses $(0.03 \mathrm{~ms}$ duration for A-fiber units or $0.3 \mathrm{~ms}$ duration for C-fiber units) through bipolar platinum electrodes. The intensity of the electrical stimulus (up to $25 \mathrm{~V}$ ) was adjusted between 1 and 1.5 times threshold for C-fiber units and twice threshold for A-fiber units.

\section{Conduction velocity (CV)}

The conduction distance (between 4.5 and $14 \mathrm{~mm}$ ) and the latency to onset of AP (evoked by dorsal root electrical stimulation) were used to estimate the $\mathrm{CV}$ of each neuron. Neurons from the two groups of rats (normal and SNA) were classified according to their dorsal root $\mathrm{CVs}$ as $\mathrm{C}(\leq 0.8 \mathrm{~m} / \mathrm{sec}), \mathrm{A} \delta(1.5-6.5 \mathrm{~m} / \mathrm{sec})$, or $\mathrm{A} \alpha / \beta$ $(>6.5 \mathrm{~m} / \mathrm{sec}) .^{21}$ The borderline between $\mathrm{A} \delta$ and $\mathrm{A} \alpha / \beta$ waves and between $\mathrm{A} \delta$ and $\mathrm{C}$ waves was determined from the findings of compound AP recordings reported previously ${ }^{21}$ in rats with the same sex, age/weight, and recording conditions as in the present experiments. The findings of those compound AP recording experiments showed that the mean value for the borderline between $\mathrm{A} \delta$ and $\mathrm{A} \alpha / \beta$ waves was $6.5 \pm 0.32 \mathrm{~m} / \mathrm{sec}$, and that there are two $\mathrm{C}$ waves, a fast wave and a slow wave. The fastest component of the slower $\mathrm{C}$ wave displayed a conduction velocity of $0.7-0.8 \mathrm{~m} / \mathrm{sec}$, whereas the fastest component of the faster wave (which we designated as a $\mathrm{C} / \mathrm{A} \delta$ wave) conducted at $1.4-1.5 \mathrm{~m} / \mathrm{sec}$. We also referred to our Review ${ }^{22}$ in which we described how $\mathrm{A} \beta$ conduction velocity is determined. We have now added this information to the Methods section of this article. These CVs were relatively low, for reasons discussed previously. ${ }^{22,23}$ These include the young age of rats, the CVs being lower in the dorsal root than in peripheral nerve fibers, inclusion of utilization time, and the low temperature in the paraffin pool.

\section{$A P$ recordings}

A Cambridge Electronics Design (CED) 1401 plus interface was used to record APs online. APs were subsequently analyzed offline using CED Spike II program as reported previously. ${ }^{19}$

\section{$A P$ variables and selection criteria}

As described previously, ${ }^{19}$ the variables measured for each neuron (see Figs. 1 and 2 and Table 1 ) included conduction velocity $(\mathrm{CV})$, resting membrane potential (RMP), AP height, AP overshoot, AP duration at base, AP rise time (RT), and AP fall time (FT). The afterhyperpolarization (AHP) variables included duration to $80 \%$ recovery (AHP 80\%) and AHP amplitude (between RMP and maximum AHP depth). Neurons were included in the analyses only if they had RMP $\leq-40 \mathrm{mV}$. A-fiber neurons were included if they had an AHP, but for C-neurons, the presence of an AHP was not required. ${ }^{24}$

It is noteworthy that changes in some of the variables included in the present analysis including RMP and RT were published in our previous studies. $^{6,25}$ They were included in the present study (larger sample of neurons) to enable comparison of directions and extents of electrophysiological changes in all variables between L5-axotoneurons and L4-RF-neurons after L5-SNA.

\section{Spontaneous activity/firing (SF) recordings}

Following recordings of evoked APs by dorsal root electrical stimulation to estimate $\mathrm{CV}$, any spontaneously occurring APs were recorded for $\sim 2 \mathrm{~min}$. Neurons showing only short-lasting (a few seconds) injury discharge (high frequency) caused by soma impalement with the microelectrode but no subsequent firing were not classified as spontaneously firing units. For all neurons, assessment of SF (ongoing stimulus-independent firing) was made before sensory testing to avoid the possibility of inducing or influencing such firing by natural search stimuli applied to the hindlimb. Neurons were classified as firing spontaneously if they showed at least one spontaneous AP during the assessment period regardless of whether or not they met the selection criteria mentioned above.

\section{Sensory receptive properties}

Using hand-held innocuous and noxious mechanical and thermal stimuli, the sensory receptive properties of non-axotomized DRG neurons were identified as described previously in rat. ${ }^{19}$ Briefly, normal L4/L5 DRG neurons, and non-axotomized L4-DRG neurons in SNA rats were classified as nociceptive/nociceptors or low threshold mechanoreceptors (LTMs). Neurons were classed as nociceptors if they responded either to noxious mechanical stimuli (applied with a needle, fine forceps, or coarse toothed forceps) only, or to both noxious mechanical and noxious heat stimuli (hot water at $50-65^{\circ} \mathrm{C}$ applied with a syringe), but failed to respond to nonnoxious mechanical stimuli.

Ipsilateral L4 nociceptive neurons with $\mathrm{C}-$, $\mathrm{A} \delta$, or $\mathrm{A} \beta$-fibers included: (1) high threshold mechanoreceptors (HTMs) that responded only to noxious mechanical stimuli or had subcutaneous receptive fields and were, therefore, not tested with a heat stimulus; 


\section{C-fiber neurons}
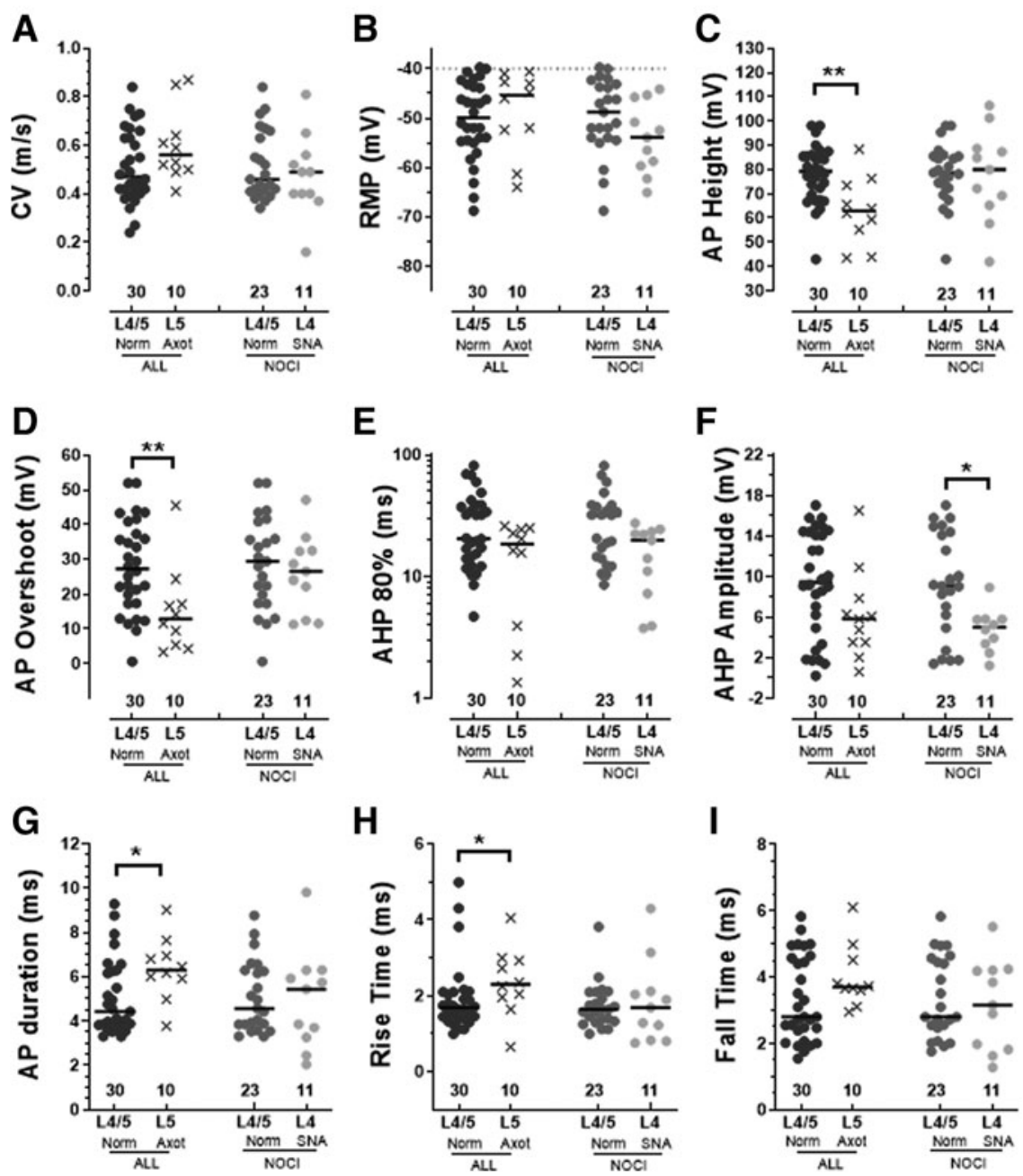

FIG. 1. Electrophysiological variables in C-fiber L5-axot-neurons and L4-RF-neurons. Scatterplots of variables measured 7 days after spinal nerve axotomy (SNA). These variables are: conduction velocity (CV) (A), resting membrane potential (RMP) (B), action potential (AP) height (C), AP overshoot (D), duration after hyperpolarization (AHP) 80\% (E), and AHP amplitude (F). The following description applies to this Figure and Figures 2-4. Each dot represents a value from one neuron. Crossed (x) symbols (second column) are L5-axot-neurons preceded by normal neurons (dotted symbols in first column) with which they are compared; symbols (fourth column) are L4-nociceptive neurons preceded by their corresponding control (normal) nocioceptive neurons. In each graph in Figures 1-3, column 2 (crossed symbols) includes all unidentified neurons (disconnected from the periphery by L5-axotomy), and column 1 includes all recorded neurons (ALL) regardless of their sensory modalities (nociceptive, non-nociceptive, and unidentified neurons) for comparison. Column 3 (dotted symbols) includes only physiologically identified L4-nociceptors (i.e., identified from their receptive fields as being nociceptors) recoded from SNA rats. Nociceptive neurons in column 4 (SNA nociceptors) are compared with their corresponding normal nociceptors in column 3 (dotted symbols) recorded from L4/L5 dorsal root ganglions (DRGs) in normal rats. The variables shown in $\mathbf{G}, \mathbf{H}$, and $\mathbf{I}$ are AP duration $(\mathbf{G})$, rise time $(\mathbf{H})$, and fall time (I). ALL: all L4/5 neurons from normal rats (Norm) regardless of sensory type (column 1); L5-AXOT: all L5 neurons recorded; these have no receptive fields because of axotomy (column 2). L4/5 Norm NOCI: all L4/5 nociceptive neurons in normal rats (column 3); L4 SNA NOCI: L4-nociceptive neurons in the ipsilateral L4 DRG after L5 SNA. Medians are superimposed. Comparisons between medians in columns 1 and 2, and between columns 3 and 4 were with the Mann-Whitney $U$ test. The level of statistical significance is as follows: $* p<0.05 ; * * p<0.01$.

and (2) mechano-heat nociceptors with superficial or dermal receptive fields that responded to noxious mechanical stimuli and also promptly to a single application of noxious heat. As we reported previously, ${ }^{19}$ DRG neurons were classified as $\mathrm{A} \beta$ nociceptors if they failed to respond to the low-intensity (nonnoxious) mechanical stimuli including light brushing of limb fur, skin contact, and light pressure with blunt objects, but responded to noxious mechanical stimuli. A $\beta$-nociceptors included "superficial cutaneous" and "deep cutaneous" units. Superficial cutaneous units were those that responded to needle pressure and pinching of the superficial skin and lifting it away from the underlying tissue with very fine forceps. These are presumed to have receptive terminals in the epidermis or the superficial dermis. Deep cutaneous units were those that did not respond to such manipulations of the superficial layers of the skin but required stimulation (squeezing) of a fold of skin, including soft dermal tissue.

In the present study, L4-neurons that were unresponsive (i.e. whose receptive fields were not found despite an extensive search 


\section{A $\delta$-fiber neurons}

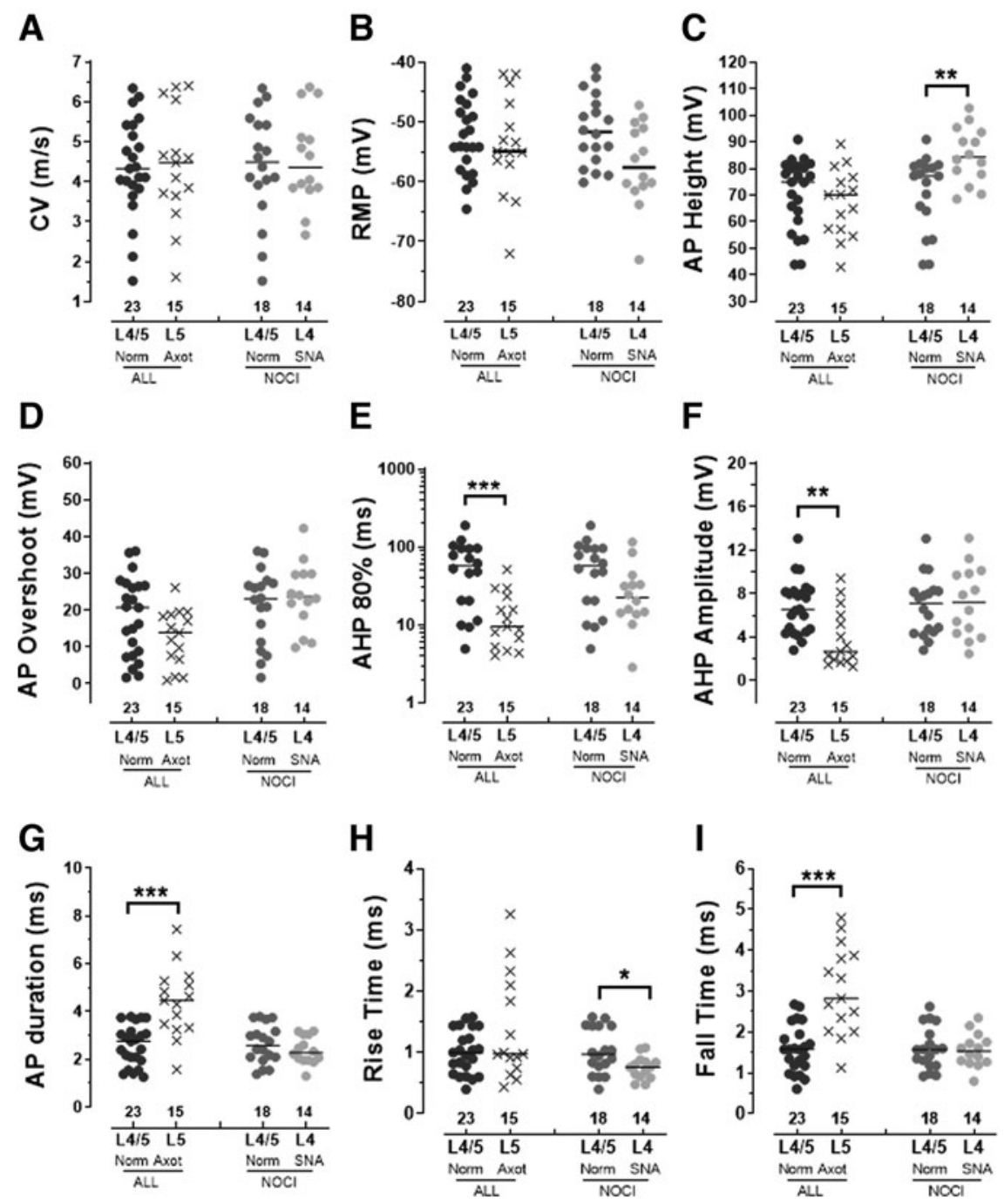

FIG. 2. Electrophysiological variables of $\mathrm{A} \delta$ L5-axot- and adjacent L4-RF-neurons. The variables shown and the details are as in Figure 1.

with all the previously describer stimuli) in normal (unoperated) rats (9 $\mathrm{C}$ - and $7 \mathrm{~A} \delta$-fiber neurons) and SNA (5 C-fiber neurons) rats were excluded from the analyses to ensure exclusion of neurons that were damaged by SNA surgery. Because we could not distinguish between silent nociceptors ${ }^{19}$ and damaged L4-neurons, we included only physiologically identified L4 neurons (nociceptors or LTMs $^{6}$ ). As described previously, ${ }^{19}$ LTMs were those units that responded to non-noxious mechanical stimuli including light brushing of limb fur with a fine paint brush, skin contact and light pressure with blunt objects, light tap, tuning forks vibrating at 100 or $250 \mathrm{~Hz}$, and pressure with calibrated von Frey hairs. LTMs conduct in all $\mathrm{CV}$ groups $(\mathrm{C}, \mathrm{A} \delta$, and $\mathrm{A} \alpha / \beta)$, but the slowly conducting $\mathrm{C}$-and $\mathrm{A} \delta$-fiber $\mathrm{LTMs},{ }^{19}$ were not encountered in the present study. Therefore, only $\mathrm{A} \beta$-LTMs were included in the current study; they were classed as slowly adapting (SA) units or rapidly adapting (RA) units including the guard $(\mathrm{G})$ hair and field (F) units that ere grouped together as G/F units. ${ }^{19} \mathrm{~A} \beta$-LTMs were further divided into cutaneous and muscle spindle afferents, but only cutaneous $\mathrm{A} \beta$-LTMs were included in the present study for comparison with nociceptors, most of which were cutaneous.

\section{Statistical analysis}

Most of the electrophysiological data were not normally distributed, and therefore non-parametric statistics were used throughout. Changes in axotomized L5-DRG neurons resulting from L5-SNA were examined as follows. For each $\mathrm{CV}$ group ( $\mathrm{C}-, \mathrm{A} \delta$-, or $\mathrm{A} \beta$-fiber), L5 axotomized neurons (no receptive fields) were compared with neurons of all types (regardless of receptive field properties) in nonaxotomized L4/5-DRGs in normal rats. Mann-Whitney tests were used for comparing the medians of the followings: (1) the variables in these two groups of neurons (columns 1 and 2 of graphs in Figs. 1-3), (2) the variables in ipsilateral $\mathrm{C}$-, $\mathrm{A} \delta$-, or $\mathrm{A} \beta$-nociceptive $\mathrm{L} 4-\mathrm{DRG}$ neurons in SNA rats (column 4 in Figs. 1-3) with those of their corresponding normal nociceptive L4/L5 neurons (columns 3 in Figs. 1-3), and (3) the variables in cutaneous L4 A $\beta$-LTM neurons in SNA rats (Fig. 4) with those of their corresponding normal (control) A $\beta$-LTM neurons. All tests were made with Graphpad Prism 8 software (Graphpad software Inc. USA). Significance for the above tests was indicated as follows $* p<0.05, * * p<0.01$, ***p $p<0.001$. Summaries of significant changes are shown in Table 1. 


\section{A $\beta$-fiber neurons}

A
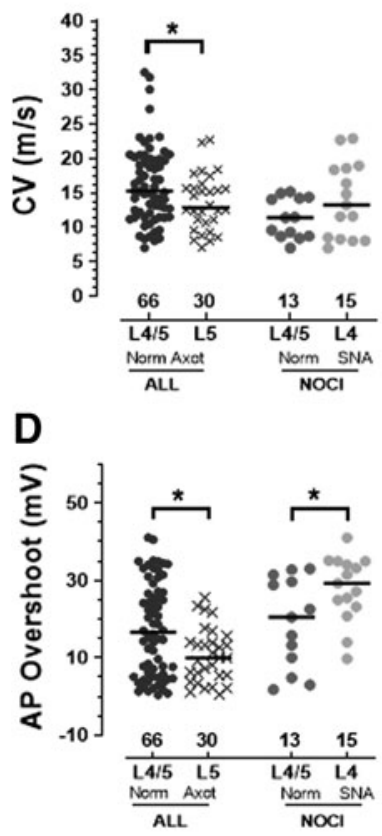

G

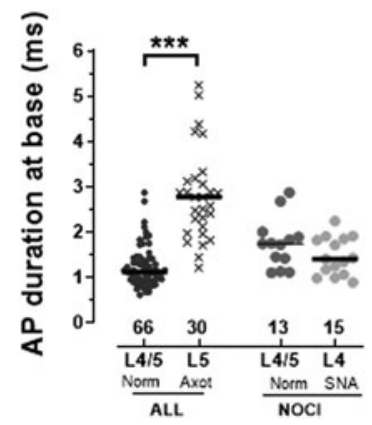

B

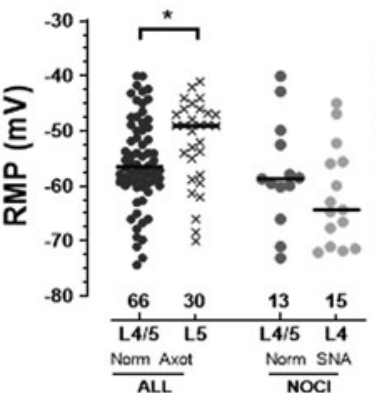

$E$

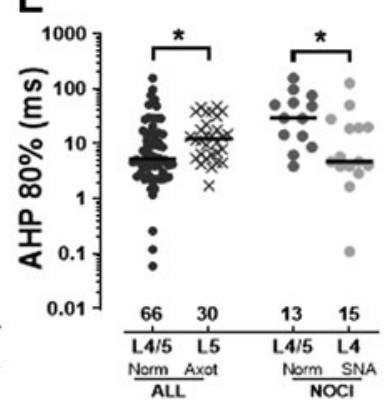

H

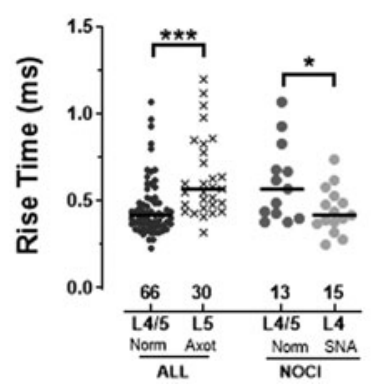

C
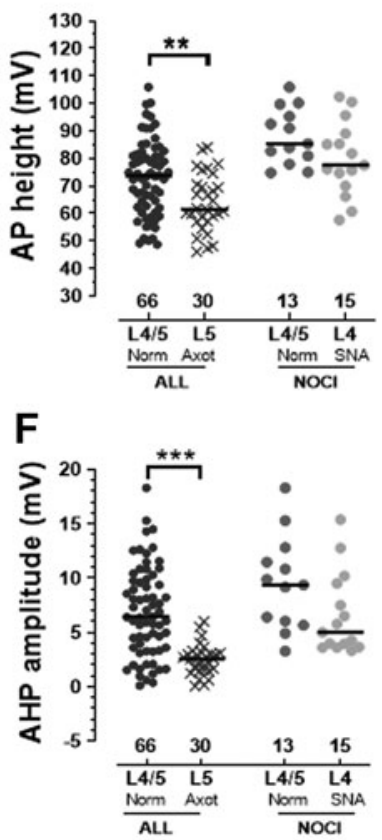

I

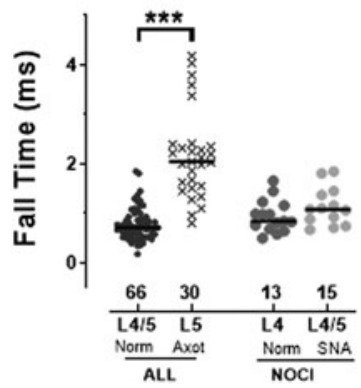

FIG. 3. Electrophysiological variables in $\mathrm{A} \beta$ L5-axot- and L4-RF-neurons. The variables shown and details are as in Figure 1.

\section{Results}

\section{SNA rats exhibit behavioral signs of mechanical, heat, and cold hypersensitivity}

As shown in Figure 5 and consistent with our previous studies, ${ }^{6,11}$ we found that all SNA rats exhibited, 7 days post-SNA, significant $(p<0.01)$ decreases in the mean PWT and in the mean PWL, indicating that they developed behavioral signs of mechanical and heat hypersensitivity respectively. We also examined whether SNA rats exhibit cold hypersensitivity. Comparison of cold sensitivity values 7 days after SNA with the baseline values (before SNA induction) showed significant increases $(p<0.01)$ in the mean duration of cold-evoked responses indicating development of cold hypersensitivity in SNA rats 7 days after SNA (Fig. 5) in agreement with previous studies. ${ }^{26}$

\section{In vivo intracellular voltage recordings in normal and SNA rats}

In vivo intracellular recordings of somatic APs were made from 251 DRG neurons in deeply anesthetized SNA rats $(n=29) 7$ days post-SNA or in normal rats $(n=43)$ of similar age/weight.
These neurons met the acceptance criteria defined in the Methods. Of these, 119 neurons were recorded from L4/L5 DRGs in normal rats (30 C-fiber, $23 \mathrm{~A} \delta$-fiber, and $66 \mathrm{~A} \beta$-fiber). Most of the normal C-fiber (23/30) and $\mathrm{A} \delta$-fiber (18/23) neurons were nociceptors, whereas most of normal $\mathrm{A} \beta$-fiber neurons $(53 / 66)$ were LTMs. Fifty-five neurons were recorded from axotomized L5 DRGs (10 C-fiber, $15 \mathrm{~A} \delta$-fiber, and $30 \mathrm{~A} \beta$-fiber) 7 days post L5-SNA; that is, they were L5-axot-neurons. The remaining 85 neurons were recorded from adjacent L4 DRGs in SNA-rats. All these L4 DRG neurons were functionally identified as nociceptors (23 C-fiber, $18 \mathrm{~A} \delta$-fiber, and $13 \mathrm{~A} \beta$-fiber) or A $\beta$-fiber LTMs (7 G hair/field $[\mathrm{G} / \mathrm{F}]$ units, $14 \mathrm{RA}$ units, and $10 \mathrm{SA}$ units). The numbers of all axotomized neurons and of functionally identified nociceptive and A $\beta$-LTM neurons in each CV group in normal and SNA groups are indicated in Figures 1-4.

In the following sections, changes in electrophysiological properties are described in relation to those of appropriate neurons in normal rats. Note that especially for $\mathrm{A} \beta$-neurons, the medians and ranges for some variables (e.g., AP duration and AHP duration) in axotomy controls (column 1) differ from those in normal nociceptors (column 3 in Figs. 1-3). This is because axotomy controls include all neurons recorded in normal rats regardless of their 
A $\beta$-fiber low threshold mechanoreceptors

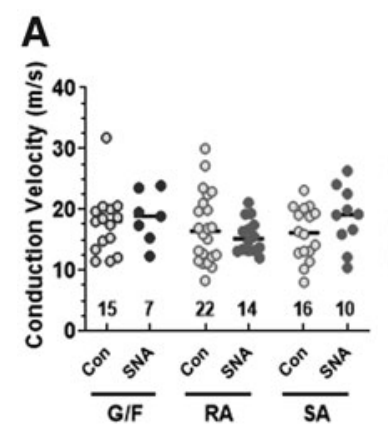

B C
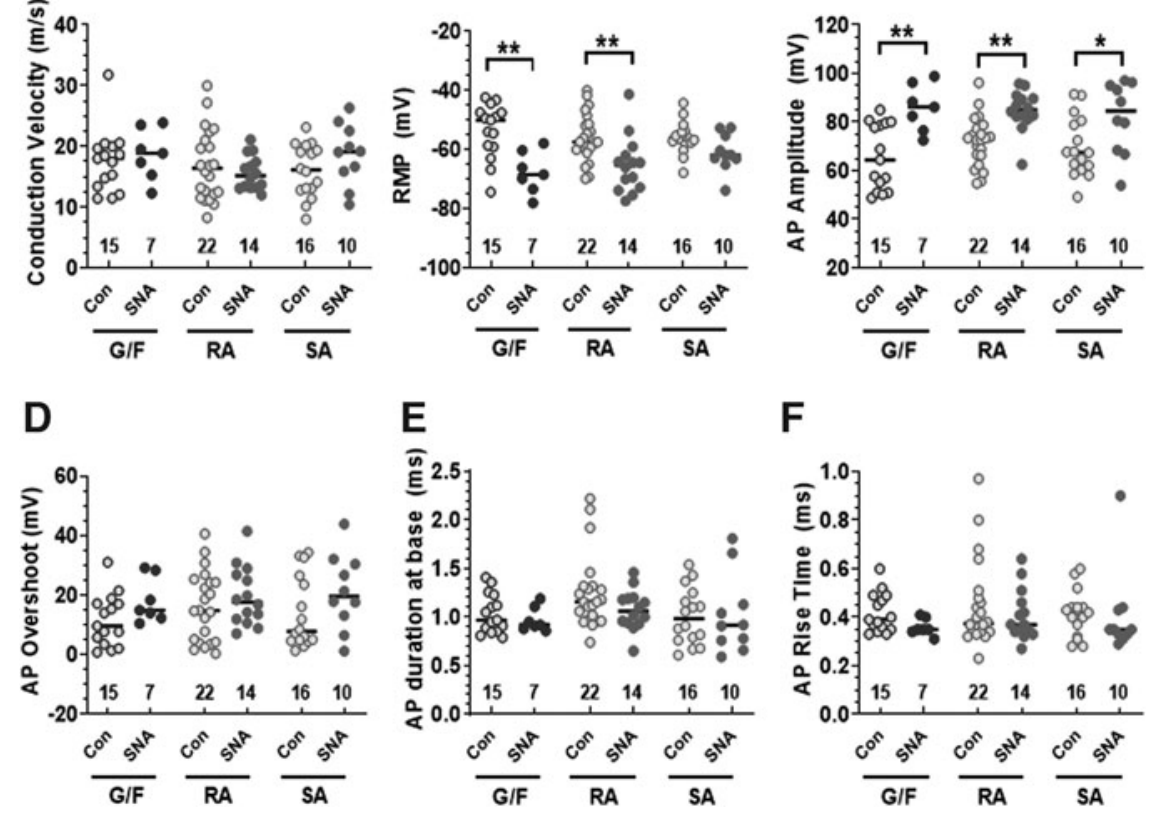

$\mathbf{F}$

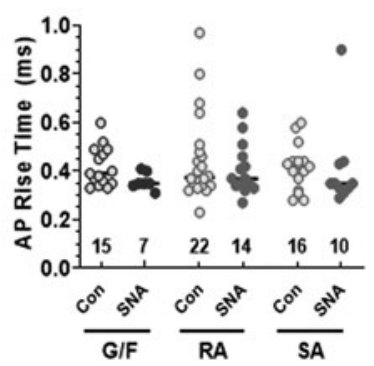

FIG. 4. Electrophysiological and action potential (AP) variables in cutaneous L4 A $\beta$-low threshold mechanoreceptors (LTMs) after L5-axotomy. The variables shown are conduction velocity (CV) (A), resting membrane potential (RMP) (B), AP amplitude (C), AP overshoot duration (D), action potential (AP) duration $(\mathbf{E})$, and AP rise time $(\mathbf{F})$. Changes in the subclasses of cutaneous A $\beta$-low threshold mechanoreceptors (LTMs) (GF, rapidly adapting [RA], and slowly adapting [SA] units) are shown separately. As in Figure 1, each dot represents one neuron, and comparisons were made between medians of the variables measured 7 days after SNA (SNA) in the subclasses of A $\beta$-LTMs and those of their respective normal (control). Con: control; G/F: G hair/field units; RA: rapidly adapting units; SA: slowly adapting units. All comparisons were made with the Mann-Whitney $U$ test. The level of statistical significance is as follows: $* p<0.05 ; * * p<0.01$.

sensory modality, and most of them are LTMs. It is noteworthy that $\sim 80 \%$ of $\mathrm{A} \beta$-neurons ${ }^{23}$ are LTMs with electrophysiological properties that differ from those of nociceptors. ${ }^{19}$

Typical examples of somatic APs in normal nociceptors, L5axot-neurons, and L4 nociceptors in SNA are shown in Figure 6 which illustrates the findings shown in Figs. 1-3, that APs in L5-axot-neurons tend to be broader in all CV groups (both $\mathrm{C}$ and A-neurons) than normal neurons. In contrast, APs in uninjured conducting L4-nociceptors are narrower with faster kinetics than those in normal rats (see Figs. 1-3). Scatterplot distributions of the measured variables are given separately for C- (Fig. 1), A $\delta$ (Fig. 2), and $\mathrm{A} \alpha / \beta$-neurons (Fig. 3). Figure 4 shL5-axot-neurons ows the distribution of the variables in subgroups of cutaneous A $\beta$-LTMs. Results will be described for first and then for L4-nociceptors, in the order of the $\mathrm{CV}$ groups: $\mathrm{C}-, \mathrm{A} \delta$-, and $\mathrm{A} \beta$ neurons (Figs. 1-3). This will be followed by a description of the changes in L4-A $\beta$-LTMs (Fig. 4). Summaries of the findings are given in Table 1.

\section{A}

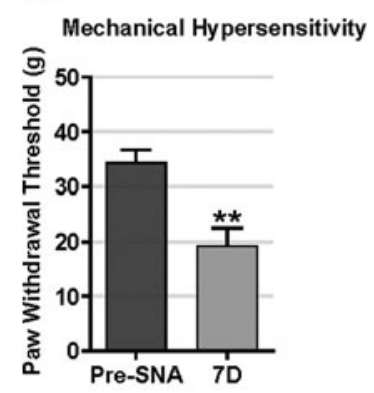

B

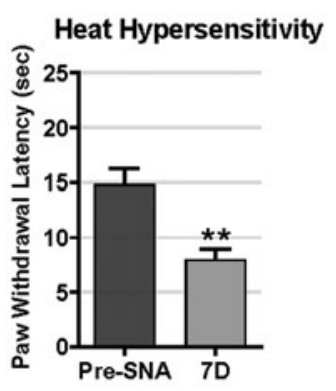

C

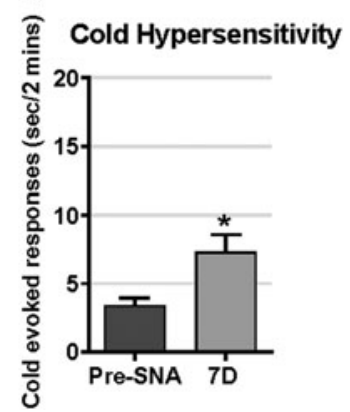

FIG. 5. Behavioral indices of mechanical, heat and cold hypersensitivity in spinal nerve axotomy (SNA) rats. Data are presented as mean \pm standard error of the mean (SEM). SNA rats exhibited significant decreases in the mean paw withdrawal threshold (A), the mean paw withdrawal latency (B), and the mean duration of cold-evoked responses $(\mathbf{C})$ 7days post-SNA indicating that the rats $(n=10)$ that underwent SNA surgery developed mechanical, heat, and cold hypersensitivity, respectively. Comparisons were between values before SNA induction (pre-SNA, dark gray bars) and 7 weeks post-SNA (7D, lighter gray bars). Statistical tests were made with paired $t$ test. The levels of statistical significance are $* p<0.05$ and $* * p<0.01$. 
A
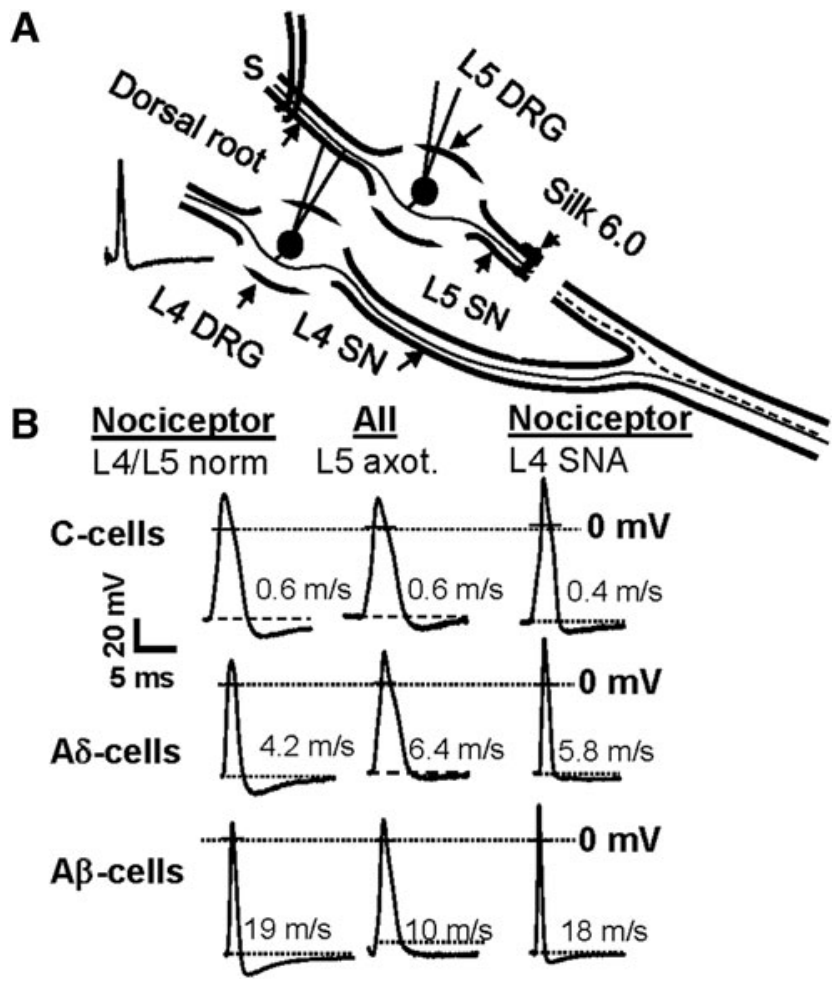

FIG. 6. Diagram of experimental setup and examples of somatic action potentials (APs). (A) The L5 spinal nerve was ligated (with 6.0 silk suture) and transected; the L4 spinal nerve was untreated. Intracellular recordings of APs were made from the somata of L5-axot.neurons in L5 dorsal root ganglions (DRGs) and adjacent L4-RF-neurons in L4 DRGs 7 days post-SNA as well as normal L5/L4 DRG neurons. APs were evoked antidromically by dorsal root electrical stimulation (S). (B) Examples of these APs recorded intracellularly from $\mathrm{C}$ - (top panel), $\mathrm{A} \delta$ - (middle panel), and $\mathrm{A} \beta$ fiber neurons (bottom panel). Column 1: normal nociceptive neurons (L4/L5 Norm); column 2: L5-axot-neurons (L5 axot.); and column 3: L4-nociceptive neurons from SNA-rats (L4 SNA). APs are selected to represent the median values of AP duration at base, afterhyperpolarization (AHP) amplitude, and AP overshoot. The conduction velocity $(\mathrm{CV})(\mathrm{m} / \mathrm{sec})$ of each neuron is given. Note the increased AP duration and decreased AHP amplitude in axotomized L5 $\mathrm{A} \delta$ - and $\mathrm{A} \beta$-neurons after L5-SNA (second column).

\section{Spontaneous firing in SNA rats}

As previously reported, ${ }^{6,11,20} \mathrm{C}$ - and A-fiber neurons fired spontaneously in SNA rats. For C-nociceptors, $\sim 4 \%$ of neurons exhibited SA in normal rats, but this was reduced to $0 \%$ in the L5 DRGs after axotomy, and rose to $\sim 35 \%$ in L4 DRGs in SNA rats. None $(0 \%)$ of the $\mathrm{A} \delta$ - or $\mathrm{A} \beta$-nociceptors fired spontaneously in normal rats, but $\sim 29 \%$ of $\mathrm{L} 4 \mathrm{~A} \delta$-nociceptors and $\sim 13 \%$ of L4 A $\beta$-nociceptors fired spontaneously in SNA rats. As for the axotomized A-fiber neurons in the L5 DRG, 20\% of A $\delta$-fiber neurons and $\mathrm{A} \beta$-fiber neurons fired spontaneously in SNA rats. It should be noted that these values of SF incidence are for different subtypes of DRG neurons regardless of whether or not they met the selection criteria mentioned in the Methods.

\section{Changes in axotomized L5-neurons (L5-axot-neurons)}

The comparisons that follow are between data in columns 1 and 2 in Figures 1-3.
Changes in axotomized L5 C-fiber neurons. After L5SNA, the median RMP of axotomized C-fiber neurons $(-45.41 \mathrm{mV})$, was more depolarized (by $\sim 5 \mathrm{mV}$ ) than that of normal C-fiber neurons $(-49.89 \mathrm{mV})$. As shown in Figure $1 \mathrm{~B}$, and unlike in our previous study, ${ }^{25}$ this change in RMP did not reach statistical significance. However, the median AP height and AP overshoot in axotomized C-fiber neurons was significantly $(p<0.01)$ lower than that in normal $\mathrm{C}$-fiber neurons (Fig.1 $\mathrm{C}$ and D). The other AP variables: median CV, AHP 80\% and AHP amplitude shown in Figure 1A, E, and F respectively exhibited no significant changes. The median AP duration increased significantly (Fig. 1G); this was largely because of a significant increase in RT (Fig. 1H) but not in FT (Fig. 1I).

Changes in axotomized L5A $\delta$-fiber neurons. In axotomized L5 A $\delta$-fiber neurons, several variables changed significantly compared with normal A $\delta$-fiber neurons (Fig. 2). Their median AHP 80\% duration and AHP depth decreased significantly (Fig. 2E and F), but there were no significant changes in the other variables shown in Figure 2 A-D. The AP duration at base (Fig. 2G) and AP FT (Fig. 2I) were both highly significantly increased, but with no change in the AP RT (Fig. 2H). Thus, the AP kinetics of axotomized L5 A $\delta$-fiber neurons were reduced substantially.

Changes in axotomized L5A $\beta$-fiber neurons. Interestingly, axotomized L5 A $\beta$-neurons exhibited significant changes in all measured variables (Fig. 3) particularly in AHP depth, AP duration at base, RT, and FT, which were all highly significantly $(p<0.001)$ different from those in normal neurons. Simply, the APs were shorter and broader with slower kinetics, and with a much less pronounced AHP (Fig. 2). Therefore, the impact of L5-SNA on large myelinated $\mathrm{A} \beta$-fiber neurons was very marked.

\section{Changes in L4-nociceptive neurons}

As described in the Methods section, the data from intact nociceptive neurons in L4 DRGs after L5-SNA, shown in column 4 (L4 SNA, red dots) were compared with column 3 (controls, purple dots) in Figures 1-3. The comparisons are to assess whether the changes in uninjured L4 nociceptors (L4-RFneurons) are in the same or in the opposite direction from those in the L5-axot-neurons.

Changes in L4 C-fiber nociceptive neurons. Compared with normal C-nociceptors, there was no significant change in any of the variables in the L4 C-fiber nociceptors measured after the L5 SNA (Fig. 1) except AHP amplitude that decreased significantly (Fig. 1F). As shown in Figure 1, there was no significant change in the AP variables (AP duration, RT, height and overshoot) that changed significantly in axotomized L5 C-nociceptors. This indicates that the slowing of AP kinetics seen in axotomized C-fiber neurons (L5-axot-neurons) does not occur in C-fiber nociceptors (L4-RF-neurons).

Changes in $\mathrm{L} 4 \mathrm{~A} \delta$-fiber nociceptors. There were no statistical differences between normal L4 $\mathrm{A} \delta$-nociceptors and uninjured L4 A $\delta$-nociceptors after L5-SNA (columns 3 and 4, Fig. 2), except for a significant increase $(p<0.01)$ in AP height (Fig. 2C) and a significant decrease in AP RT (Fig. 2H). There was no significant change in these variables in axotomized $\mathrm{A} \delta$-fiber neurons (Fig. 2), which supports our hypothesis that the changes in L5-axot-neurons and L4-RF-neurons are distinct. 
Changes in L4 A $\beta$-fiber nociceptors. L4 A $\beta$-fiber nociceptors in SNA rats exhibited a significant increase $(p<0.05)$ in the AP overshoot (Fig. 3D) and a significant decrease $(p<0.05)$ in AHP $80 \%$ duration (Fig. 3E). Interestingly, as shown in Figure 3D and E, the changes in these variables were in the opposite directions from those in axotomized $\mathrm{L} 5 \mathrm{~A} \beta$-fiber neurons.

\section{Changes in L5-axot-neurons versus intact L4-RF-nociceptors}

Interestingly, most of the variables that changed significantly or tended to change in nociceptive L4-neurons after L5-SNA were in the opposite directions from those in axotomized L5-neurons. These include AP overshoot (Fig. 3D), AHP duration (Fig. 3E), and AP RT (Fig. $3 \mathrm{H}$ ) in A $\beta$-fiber neurons. The opposite changes in A $\beta$ fiber neurons may be expected if these changes were dependent on factors derived from the periphery (see Discussion).

\section{Changes in cutaneous L4 $\beta$-LTM neurons}

We also examined whether the variables mentioned also change in cutaneous L4 A $\beta$-LTMs after L5 SNA. As shown in Figure 4B, both G/F units and RA units had a more hyperpolarized median RMP ( $p<0.01$ in both cases) than their respective normal groups. Both of these subtypes (G/F and RA) and SA units also exhibited significantly greater AP amplitude than their respective controls (Fig. 4C). There were no significant changes in the variables shown in Figure 4 or other variables (not shown) in cutaneous L4 A $\beta$-LTMs.

\section{Discussion}

We have found that electrophysiological changes in L5-axotneurons differ from those of adjacent L4-RF-neurons 7 days after L5-SNA. L5-axot-neurons exhibited slower AP kinetics in all CV groups (A $\alpha / \beta-, \mathrm{A} \delta$-, and $\mathrm{C}$-fiber neurons) with significant changes in all measured variables in $\mathrm{A} \beta$-fiber neurons, and several variables in $\mathrm{C}$-and $\mathrm{A} \delta$-fiber neurons (see Table 1$)$. A few variables changed significantly in L4-RF-neurons but not in L5-axot-neurons, including decreased AHP amplitude in C-fiber nociceptors and decreased RT and increased AP amplitude/height in A $\delta$-fiber nociceptors. More interestingly, all the significant changes seen in L4 A $\beta$-fiber nociceptors (L4-RF-neurons) were in the opposite direction from those in axotomized L5 A $\beta$-fiber neurons (L5-axotneurons). These opposite changes might be expected if they were dependent on trophic factors derived from the periphery (described subsequently). L4 A $\beta$-fiber LTMs also exhibited significant changes in some variables (see Fig. 4). Whether similar or different patterns of changes occur at other time points after nerve injury remains to be determined. The faster AP kinetics (decreased refractory period) in L4-RF-neurons that are essential for transmission of evoked pain signals to the CNS are consistent with their reported hyperexcitability and spontaneous firing. ${ }^{6,11}$ The faster AP kinetics in uninjured L4 neurons that may be related to increased trophic factors and neuroinflammation associated with Wallerain degeneration of axotomized L5-fibers (described subsequently) may lead to repetitive firing, and thus provide enhanced afferent input necessary for initiating and/or maintaining PNP development. It should be noted that some of the electrophysiological changes in L4-RF-neurons may also be caused by the impact of trauma at the time of SNA surgery. In contrast, the slower AP kinetics in L5axot-neurons that could result from deprivation of target-derived trophic factors such as NGF and glial cell line-derived neurotrophic factor (GDNF) (described subsequently) are consistent with the lack of spontaneous firing in axotomized C-fiber neurons reported previously. ${ }^{6,11}$ These findings in axotomized L5-neuorns together with their being disconnected from their peripheral targets suggest that they are less likely to make a significant contribution to PNP pathogenesis, although they may contribute to the central mechanisms of PNP via enhanced neurotransmitter release in the CNS.

\section{Electrophysiological changes in L5-axot-neurons after L5-SNA}

Our findings of changes in L5-axot-neurons are consistent with those of previous studies showing that damaged L5-DRG neurons have broader somatic APs with slower kinetics after L5SNL $^{9,10,27,28}$ or L5-SNA. ${ }^{8,10,29-31}$ Such changes could result from deprivation of target-derived trophic factors such as NGF and GDNF, ${ }^{32}$ causing downregulation of Nav1.8, Nav1.9, ${ }^{33,34}$ and Nav1. $7^{35}$ sodium channels. The trophic factor deprivation may well explain similarities between changes in L5-axot-neurons in vivo (present study) and those observed in previous in vitro and ex-vivo studies, where such deprivation also occurs. These similarities include decreased AP height, ${ }^{9}$ increased AP duration, ${ }^{10}$ and slower AP kinetics. ${ }^{8}$ In the present study, we found electrophysiological changes in axotomized $\mathrm{A} \beta$-neurons to be proportionally greater and more significant than those in $\mathrm{A} \delta$ - or C-neurons (see Table 1), probably because $\mathrm{A} \delta$ - and C-neurons normally have slower AP kinetics than $A \alpha / \beta$-neurons, and therefore less potential for further slowing. These findings in L5-axot-neurons are consistent with those reported by Ma and coworkers, ${ }^{8}$ who found several electrophysiological changes including broader (slower) APs in the somata of large- and medium-sized, but not small-sized neurons. Conversely, our findings are not in line with those reported previously that excised $\mathrm{A} \delta$-fiber neurons exhibited the most pronounced electrophysiological changes 20 days after SNL. ${ }^{10}$ This apparent discrepancy might be the result of the differences in the time points at which electrophysiological changes were examined (7 vs. 20 days), in the categorization of DRG neurons on the basis of dorsal root CVs, and, more importantly, in the experimental type (in vivo vs. in vitro). The borderlines between $\mathrm{CV}$ groups in our rat studies are relatively low (lower than those of Sapunar and coworkers ${ }^{10}$ ) for reasons reported previously (see Methods). In our study, neurons were classified as $\mathrm{A} \delta$-type if their dorsal root $\mathrm{CVs}$ were $>1.5 \mathrm{~m} / \mathrm{sec}$ and $<6.5 \mathrm{~m} / \mathrm{sec}$. As reported previously, ${ }^{21}$ our classification is based on compound AP recordings from L4/L5 dorsal roots of female rats of the same age/weight as used in the present study, and the same recording conditions. Interestingly the electrophysiological changes in L5-axot-neurons seen in the present study were in the same direction in all CV groups (see Table 1), consistent with common underlying mechanisms in all $\mathrm{CV}$ groups.

\section{Electrophysiological changes in uninjured L4-RF-neurons after L5-SNA}

A few previous studies examined the impact of L5-SNL/SNA on electrophysiological properties of adjacent L4-neurons in exteriorized and superfused ex vivo DRGs, ${ }^{8}$ acutely excised or cultured dissociated DRG neurons, ${ }^{9,10,27,28}$ and in vivo. ${ }^{6,16}$ Some of these studies reported changes in axotomized L5-neurons but not in L4-neurons, including AP height ${ }^{9}$ and increased AP duration. ${ }^{10}$ Of the aforementioned studies, the study with the closest methodology to ours was that in vivo by Boada and coworkers, ${ }^{16}$ who recorded from rat L4-RF-neurons 7 days after partial L5-SNL (pSNL), but as in our previous study, ${ }^{6}$ they did not record from axotomized 
L5-neurons. Their L4 neurons showed decreased AHP durations (at half-amplitude) in A-HTMs. We also found a significant decrease in AHP duration in L4 A $\beta$-nociceptors (see Table 1), but not in A-HTMs (combined $\mathrm{A} \beta$-and $\mathrm{A} \delta$-fiber nociceptors). This might be because of the differences in the type of spinal nerve injury (L5 SNA vs. partial pSNL), the animal age (young adult vs. juvenile) or rat strains (Wistar vs. Sprague-Dawley). Interestingly, Boada and coworkers ${ }^{16}$ also reported that pSNL induced sensitization of L4 AHTMs after pSNL, a sevenfold reduction in mechanical threshold of L4 A-HTMs and a sevenfold increase in their receptive fields. We and others have also previously shown that L4 A $\beta$ - nociceptors $^{36}$ and $\mathrm{L} 4 \mathrm{~A} \delta$ - and $\mathrm{C}$-fiber nociceptors ${ }^{37}$ become sensitized to mechanical and/or heat stimuli after L5-SNA/SNL.

Our current in vivo findings that significant changes in L4-nociceptors occurred only in L4-RF-neurons ( $\mathrm{C}$-and $\mathrm{A} \delta$-fiber nociceptors) or being in the opposite direction from those in axotomized L5A $\beta$-neurons (A $\beta$-nociceptors, see Table 1 ), differ from those of the aforementioned ex vivo study, ${ }^{8}$ which reported similar changes in both groups of DRG neurons after SNL. This apparent discrepancy may be because of the differences in data sampling. Indeed, it is possible that some of the similarities between L5-axot-neurons and adjacent L4-neurons reported by $\mathrm{Ma}$ and coworkers ${ }^{8}$ resulted from their inclusion of injured L4-neurons (e.g., because of L5 surgery). This is because only a few of their L4neurons were L4-RF-neurons (had identified sensory receptive properties), whereas all of our L4-neurons were L4-RF-neuron; that is, they were all physiologically identified as nociceptors or LTMs. We believe that the role of intact L4 neurons in PNP induced by L5 SNA/SNL needs to be studied in vivo because of losses in vitro of: (1) the chemical environment including peripherally derived trophic factors and/or inflammatory mediators, and (2) sensory terminals and fibers (after dissociation) preventing CV measurement. Loss of in vitro environment and damage profoundly alter neuronal properties including previously intact L4-neurons (after L5 SNA/SNL), and normal (untreated) neurons, thus altering normal values against which comparisons are measured. ${ }^{38}$ In vivo influences on intact L4-neurons after L5-SNA/SNL may include increased inflammatory mediators (e.g., tumor necrosis factor [TNF]- $\alpha$ and other cytokines), and availability/transport of NGF to their somata, ${ }^{14}$ plus lowered $\mathrm{pH}$. NGF and inflammation have profound effects on DRG neurons. For example, we have previously shown that increases in AP kinetics and spontaneous firing in $\mathrm{C}$ - and $\mathrm{A} \delta$-nociceptors induced by persistent inflammation were NGF dependent. ${ }^{39}$ Therefore, some of the changes in intact L4 nociceptors reported here may depend on raised NGF. TNF- $\alpha$ triggers ongoing firing in DRG neurons especially after nerve injury $^{40,41}$ and reduces mechanical thresholds of afferent fibers. ${ }^{42}$ These findings suggest that other inflammatory mediators such as TNF- $\alpha$ may also contribute to the electrophysiological properties in L4-RF-neurons after SNL/SNA. Neuroinflammatory mediators may directly activate fibers of L4 neurons and/or be transported to somata of these neurons causing long-term changes in receptor or ion channel expression/activation and thus membrane properties (for reviews Watkins and Maier ${ }^{43}$ ).

\section{Possible ionic mechanisms of the changes in axotomized L5-and adjacent L4-neurons}

The changes in AP variables (AP height, overshoot, duration, RT and all time) in L5-axot-neurons including the decreases in AP height and $\mathrm{AP}$ overshoot in $\mathrm{C}$-and $\mathrm{A} \beta$-fiber neurons are likely to be caused by decreased expression in voltage-gated $\mathrm{Na}^{+}$(Nav) chan- nels. This is because: (1) these related variables are dependent on expression of Nav1.8 that carries the majority of the AP inward current, ${ }^{44,45}$ (2) Nav1.8 is correlated with AP overshoot in Nav1.8 positive DRG neurons, ${ }^{46}$ and (3) expression of Nav1.7 and Nav1.8 channels and the tetrodotoxin (TTX)-resistant $\mathrm{Na}^{+}$current is decreased in axotomized DRG neurons. ${ }^{47-50}$ The increased duration of AP fall time in axotomized L5 A $\beta$-and A $\delta$-fiber neurons (L5axot-neurons) which is often associated with an inflection/hump on AP falling phase of some neurons may result from reduced TTXresistant $\mathrm{Na}^{+}$currents. ${ }^{51}$ Calcium activated channels $\left(\mathrm{Ca}_{\mathrm{v}}\right)$ may also contribute because $\mathrm{Ca}^{2+}$ contributes a large inward current $\left(I_{C a}\right)$ during the AP repolarization phase, leading to a prolongation or inflection of the descending limb, whereas removing $\mathrm{Ca}^{2+}$ shortens AP duration. ${ }^{52}$ Direct evidence for the role of $\mathrm{Ca}_{\mathrm{v}}$ channels in the AP prolongation in L5-axot-neurons was provided more recently by McCallum and coworkers ${ }^{27}$ who found that $I_{C a}$ decreased substantially in small- and medium-sized L5-neurons after SNL, and that $I_{C a}$ losses were associated with AP prolongation in these neurons. They also found that $\mathrm{Ca}^{2+}$ channel blockade with antagonists significantly prolonged AP duration and reduced $I_{C a}$. Based on these findings, they concluded that AP prolongation in L5-axotneurons is attributable to the loss of $I_{C a}{ }^{27}$ Calcium activated $\mathrm{k}^{+}$ channels (Kca) that contribute to the AP repolarization and duration $^{53}$ are unlikely to contribute to the increased AP duration in L5-axot-neurons, because blockers of $\mathrm{Ca}^{2+-}$ activated BK (largeconductance) and SK (small-conductance) channels had no consistent effect on AP duration in mouse DRG neurons. ${ }^{54}$

We have previously reported a significant depolarization in RMP in axotomized C-neurons. ${ }^{25}$ Such RMP depolarization did not reach statistical significance in our current sample of axotomized C-neurons probably because of the difference in the sample sizes between the two studies. Depolarized RMP in axotomized C-fiber neurons may result from loss of two pore domain $\mathrm{K}^{+}$channel (K2P) expression/activity because these channels are the greatest contributor to their RMP and because of axotomy-induced loss of membrane-associated-TREK2 in axotomized C-fiber neurons. ${ }^{25}$ Changes in expression and/or activation properties of other channels/currents such as $I h,{ }^{55,56} \mathrm{M}$ currents, ${ }^{57}$ and/or Nav1.9 ${ }^{21}$ may also contribute. The decreased AHP amplitude in axotomized A $\beta$ and $\mathrm{A} \delta$-fiber neurons could result from axotomy-induced decreased $\mathrm{K}^{+}$currents. ${ }^{58} \mathrm{Ca}^{2+-}$ activated BK and SK channels that conduct the currents underlying the fast AHP and the medium and slow AHP respectively ${ }^{59,60}$ are likely to contribute to the decreased AHP amplitude and duration seen in axotomized $\mathrm{A} \delta$-fiber neurons. This is because all $I K_{C a}$ subtypes that are mediated by different $\mathrm{K}_{\mathrm{ca}}$ channels were found to decrease in axotomized medium-and smallsized DRG neurons, which also exhibited a decrease in these AHP parameters after L5 SNL. ${ }^{27,28}$

As for the changes seen in L4-RF-neuorns (see Table 1 and Figs. 1-3), including the increased AP height in A $\delta$-fiber nociceptors and cutaneous L4 A $\beta$-LTMs, increased AP overshoot in $\mathrm{A} \beta$-fiber nociceptors, and decreased AP rise time in $\mathrm{A} \delta$-and $\mathrm{A} \beta$ fiber nociceptors, they may be related to the upregulation of Nav1.7 and Nav1.8 and increased $\mathrm{Na}^{+}$current density, which has been reported in L4 DRG neurons after L5 SNL/SNA. ${ }^{61-65}$

\section{Conclusion}

The changes in membrane properties of L5-axot-neurons induced by the L5-SNA generally differed from those of L4-RFneurons, with most of the changes occurring in L5-axot-neurons but not L4-RF-neurons or occurring in the opposite direction in the two 
populations of DRG neurons. Our findings that most changes, in the two groups of DRG neurons, are in the opposite direction are consistent with decreases of the neurotrophic influences "hypotrophy" on L5-axot-neurons and increases of the trophic factors "hypertrophy" or inflammatory mediators on intact L4-RFneurons, respectively. As noted, the changes in L5-axot-neurons and adjacent L4-RF-neurons are likely to contribute differentially to mechanisms of PNP.

\section{Acknowledgments}

We thank staff at Biomedical Services Unit (BSU) of the University of Liverpool for their technical support and help.

\section{Funding Information}

This research work was supported by a Medical Research Council grant (G0700420) and a PhD studentship from the Biotechnology and Biological Sciences Research Council to Laiche Djouhri.

\section{Author Disclosure Statement}

No competing financial interests exist.

\section{References}

1. Colloca, L., Ludman, T., Bouhassira, D., Baron, R., Dickenson, A.H., Yarnitsky, D., Freeman, R., Truini, A., Attal, N., Finnerup, N.B., Eccleston, C., Kalso, E., Bennett, D.L., Dworkin, R.H., and Raja, S.N. (2017). Neuropathic pain. Nat. Rev. Dis. Primers 3, 17002.

2. Bouhassira, D., and Attal, N. (2016). Translational neuropathic pain research: a clinical perspective. Neuroscience 338, 27-35.

3. Jensen, T.S., and Finnerup, N.B. (2014). Allodynia and hyperalgesia in neuropathic pain: clinical manifestations and mechanisms. Lancet Neurol. 13, 924-935.

4. Kim, S.H., and Chung, J.M. (1992). An experimental model for peripheral neuropathy produced by segmental spinal nerve ligation in the rat. Pain 50, 355-363.

5. Costigan, M., Scholz, J., and Woolf, C.J. (2009). Neuropathic pain: a maladaptive response of the nervous system to damage. Annu. Rev. Neurosci. 32, 1-32.

6. Djouhri, L., Fang, X., Koutsikou, S., and Lawson, S.N. (2012). Partial nerve injury induces electrophysiological changes in conducting (uninjured) nociceptive and nonnociceptive DRG neurons: Possible relationships to aspects of peripheral neuropathic pain and paresthesias. Pain 153, 1824-1836.

7. Griffin, JW. (2006). The roles of growth factors in painful lengthdependent axonal neuropathies, in: Emerging Strategies for the Treatment of Neuropathic Pain. J.N. Campbell, A.I. Basbaum, A. Dray, R. Dubner, R.H. Dworkin, C.N. Sang (eds.). Pain. IASP Press: Seattle pps. 271-290.

8. Ma, C., Shu, Y., Zheng, Z., Chen, Y., Yao, H., Greenquist, K.W., White, F.A. and LaMotte, R.H. (2003). Similar electrophysiological changes in axotomized and neighboring intact dorsal root ganglion neurons. J. Neurophysiol. 89, 1588-1602.

9. Zhang, X.F., Zhu, C.Z., Thimmapaya, R., Choi, W.S., Honore, P., Scott, V.E., Kroeger, P.E., Sullivan, J.P., Faltynek, C.R., Gopalakrishnan, M., and Shieh, C.C. (2004). Differential action potentials and firing patterns in injured and uninjured small dorsal root ganglion neurons after nerve injury. Brain Res. 1009, 147-158.

10. Sapunar, D., Ljubkovic, M., Lirk, P., McCallum, J.B., and Hogan, Q.H. (2005). Distinct membrane effects of spinal nerve ligation on injured and adjacent dorsal root ganglion neurons in rats. Anesthesiology 103, 360-376.

11. Djouhri, L., Koutsikou, S., Fang, X., McMullan, S., and Lawson, S.N. (2006). Spontaneous pain, both neuropathic and inflammatory, is related to frequency of spontaneous firing in intact $\mathrm{C}$-fiber nociceptors. J. Neurosci. 26, 1281-1292.

12. Obata, K., Yamanaka, H., Fukuoka, T., Yi, D., Tokunaga, A., Hashimoto, N., Yoshikawa, H., and Noguchi, K. (2003). Contribution of injured and uninjured dorsal root ganglion neurons to pain behavior and the changes in gene expression following chronic constriction injury of the sciatic nerve in rats. Pain 101, 65-77.

13. Shortland, P.J., Baytug, B., Krzyzanowska, A., McMahon, S.B., Priestley, J.V., and Averill, S. (2006). ATF3 expression in L4 dorsal root ganglion neurons after L5 spinal nerve transection. Eur. J. Neurosci. 23, 365-373.

14. Obata, K., Yamanaka, H., Dai, Y., Mizushima, T., Fukuoka, T., Tokunaga, A., Yoshikawa, H., and Noguchi, K. (2004). Contribution of degeneration of motor and sensory fibers to pain behavior and the changes in neurotrophic factors in rat dorsal root ganglion. Exp. Neurol. 188, 149-160.

15. Fukuoka, T., Kondo, E., Dai, Y., Hashimoto, N., and Noguchi, K. (2001). Brain-derived neurotrophic factor increases in the uninjured dorsal root ganglion neurons in selective spinal nerve ligation model. J. Neurosci. 21, 4891-4900.

16. Boada, M.D., Gutierrez, S., Aschenbrenner, C.A., Houle, T.T., Hayashida, K., Ririe, D.G., and Eisenach, J.C. (2015). Nerve injury induces a new profile of tactile and mechanical nociceptor input from undamaged peripheral afferents. J. Neurophysiol. 113, 100-109.

17. Liu, C.N., Wall, P.D., Ben-Dor, E., Michaelis, M., Amir, R., and Devor, M. (2000). Tactile allodynia in the absence of C-fiber activation: altered firing properties of DRG neurons following spinal nerve injury. Pain 85, 503-521.

18. Villiere, V., and McLachlan, E.M. (1996). Electrophysiological properties of neurons in intact rat dorsal root ganglia classified by conduction velocity and action potential duration. J. Neurophysiol 76, 1924-1941.

19. Fang, X., McMullan, S., Lawson, S.N., and Djouhri, L. (2005). Electrophysiological differences between nociceptive and nonnociceptive dorsal root ganglion neurones in the rat in vivo. J. Physiol. 565, 927-943.

20. Djouhri, L., Smith, T., Ahmeda, A., Alotaibi, M., and Weng, X. (2018). Hyperpolarization-activated cyclic nucleotide-gated channels contribute to spontaneous activity in L4 C-fiber nociceptors, but not Abeta-non-nociceptors, after axotomy of L5-spinal nerve in the rat in vivo. Pain 159, 1392-1402.

21. Fang, X., Djouhri, L., Black, J.A., Dib-Hajj, S.D., Waxman, S.G., and Lawson, S.N. (2002). The presence and role of the tetrodotoxinresistant sodium channel $\mathrm{Na}(\mathrm{v}) 1.9(\mathrm{NaN})$ in nociceptive primary afferent neurons. J. Neurosci. 22, 7425-7433.

22. Djouhri, L., and Lawson, S.N. (2001). Increased conduction velocity of nociceptive primary afferent neurons during unilateral hindlimb inflammation in the anaesthetised guinea-pig. Neuroscience 102, 669-679.

23. Djouhri, L., and Lawson, S.N. (2004). Abeta-fiber nociceptive primary afferent neurons: a review of incidence and properties in relation to other afferent A-fiber neurons in mammals. Brain Res. Brain Res. Rev. 46, 131-145.

24. Djouhri, L., Bleazard, L., and Lawson, S.N. (1998). Association of somatic action potential shape with sensory receptive properties in guinea-pig dorsal root ganglion neurones. J. Physiol. 513 ( Pt 3), 857-872.

25. Acosta, C., Djouhri, L., Watkins, R., Berry, C., Bromage, K., and Lawson, S.N. (2014). TREK2 expressed selectively in IB4-binding C-fiber nociceptors hyperpolarizes their membrane potentials and limits spontaneous pain. J. Neurosci. 34, 1494-1509.

26. Clifford, J.L., Mares, A., Hansen, J., and Averitt, D.L. (2015). Preemptive perineural bupivacaine attenuates the maintenance of mechanical and cold allodynia in a rat spinal nerve ligation model. BMC Anesthesiol. 15, 135.

27. McCallum, J.B., Kwok, W.M., Sapunar, D., Fuchs, A. and Hogan, Q.H. (2006). Painful peripheral nerve injury decreases calcium current in axotomized sensory neurons. Anesthesiology 105, 160-168.

28. Sarantopoulos, C.D., McCallum, J.B., Rigaud, M., Fuchs, A., Kwok, W.M., and Hogan, Q.H. (2007). Opposing effects of spinal nerve ligation on calcium-activated potassium currents in axotomized and adjacent mammalian primary afferent neurons. Brain Res. 1132, 84-99.

29. Abdulla, F.A., and Smith, P.A. (2001). Axotomy- and autotomyinduced changes in the excitability of rat dorsal root ganglion neurons. J. Neurophysiol. 85, 630-643.

30. Stebbing, M.J., Eschenfelder, S., Habler, H.J., Acosta, M.C., Janig, W., and McLachlan, E.M. (1999). Changes in the action potential in sensory neurones after peripheral axotomy in vivo. Neuroreport 10 , 201-206. 
31. Kim, Y.I., Na, H.S., Kim, S.H., Han, H.C., Yoon, Y.W., Sung, B., Nam, H.J., Shin, S.L., and Hong, S.K. (1998). Cell type-specific changes of the membrane properties of peripherally-axotomized dorsal root ganglion neurons in a rat model of neuropathic pain. Neuroscience $86,301-309$.

32. Priestley, J.V., Michael, G.J., Averill, S., Liu, M., and Willmott, N. (2002). Regulation of nociceptive neurons by nerve growth factor and glial cell line derived neurotrophic factor. Can. J. Physiol. Pharmacol. 80, 495-505.

33. Fjell, J., Cummins, T.R., Dib-Hajj, S.D., Fried, K., Black, J.A., and Waxman, S.G. (1999). Differential role of GDNF and NGF in the maintenance of two TTX-resistant sodium channels in adult DRG neurons. Brain Res. Mol. Brain Res. 67, 267-282.

34. Gould, H.J., 3rd, Gould, T.N., England, J.D., Paul, D., Liu, Z.P., and Levinson, S.R. (2000). A possible role for nerve growth factor in the augmentation of sodium channels in models of chronic pain. Brain Res. 854, 19-29.

35. Kim, C.H., Oh, Y., Chung, J.M., and Chung, K. (2001). The changes in expression of three subtypes of TTX sensitive sodium channels in sensory neurons after spinal nerve ligation. Brain Res. Mol. Brain Res. 95, 153-161.

36. Djouhri, L. (2016). L5 spinal nerve axotomy induces sensitization of cutaneous L4 Abeta-nociceptive dorsal root ganglion neurons in the rat in vivo. Neurosci. Lett. 624, 72-77.

37. Shim, B., Kim, D.W., Kim, B.H., Nam, T.S., Leem, J.W., and Chung, J.M. (2005). Mechanical and heat sensitization of cutaneous nociceptors in rats with experimental peripheral neuropathy. Neuroscience 132, 193-201.

38. Kerekes, N., Landry, M., Lundmark, K., and Hokfelt, T. (2000). Effect of NGF, BDNF, bFGF, aFGF and cell density on NPY expression in cultured rat dorsal root ganglion neurones. J. Auton. Nerv. Syst. 81, $128-138$.

39. Djouhri, L., Dawbarn, D., Robertson, A., Newton, R., and Lawson, S.N. (2001). Time course and nerve growth factor dependence of inflammation-induced alterations in electrophysiological membrane properties in nociceptive primary afferent neurons. J. Neurosci. 21, 8722-8733.

40. Schafers, M., Lee, D.H., Brors, D., Yaksh, T.L., and Sorkin, L.S. (2003). Increased sensitivity of injured and adjacent uninjured rat primary sensory neurons to exogenous tumor necrosis factor-alpha after spinal nerve ligation. J. Neurosci. 23, 3028-3038.

41. Sorkin, L.S., Xiao, W.H., Wagner, R., and Myers, R.R. (1997). Tumour necrosis factor-alpha induces ectopic activity in nociceptive primary afferent fibres. Neuroscience 81, 255-262.

42. Ozaktay, A.C., Kallakuri, S., Takebayashi, T., Cavanaugh, J.M., Asik, I., DeLeo, J.A., and Weinstein, J.N. (2006). Effects of interleukin-1 beta, interleukin-6, and tumor necrosis factor on sensitivity of dorsal root ganglion and peripheral receptive fields in rats. Eur. Spine J. 15, 1529-1537.

43. Watkins, L.R., and Maier, S.F. (2002). Beyond neurons: evidence that immune and glial cells contribute to pathological pain states. Physiol. Rev. 82, 981-1011.

44. Renganathan, M., Cummins, T.R., and Waxman, S.G. (2001). Contribution of $\mathrm{Na}(\mathrm{v}) 1.8$ sodium channels to action potential electrogenesis in DRG neurons. J. Neurophysiol. 86, 629-640.

45. Blair, N.T. and Bean, B.P. (2002). Roles of tetrodotoxin (TTX)sensitive Na+ current, TTX-resistant $\mathrm{Na}+$ current, and $\mathrm{Ca} 2+$ current in the action potentials of nociceptive sensory neurons. J. Neurosci. 22, $10277-10290$.

46. Djouhri, L., Fang, X., Okuse, K., Wood, J.N., Berry, C.M., and Lawson, S.N. (2003). The TTX-resistant sodium channel Nav1.8 (SNS/PN3): expression and correlation with membrane properties in rat nociceptive primary afferent neurons. J. Physiol. 550, 739-752.

47. Dib-Hajj, S.D., Tyrrell, L., Black, J.A., and Waxman, S.G. (1998) $\mathrm{NaN}$, a novel voltage-gated $\mathrm{Na}$ channel, is expressed preferentially in peripheral sensory neurons and down-regulated after axotomy. Proc. Natl. Acad. Sci. U. S. A. 95, 8963-8968.

48. Decosterd, I., Ji, R.R., Abdi, S., Tate, S., and Woolf, C.J. (2002). The pattern of expression of the voltage-gated sodium channels $\mathrm{Na}(\mathrm{v}) 1.8$ and $\mathrm{Na}(\mathrm{v}) 1.9$ does not change in uninjured primary sensory neurons in experimental neuropathic pain models. Pain 96, 269-277.

49. Cummins, T.R., and Waxman, S.G. (1997). Downregulation of tetrodotoxin-resistant sodium currents and upregulation of a rapidly repriming tetrodotoxin-sensitive sodium current in small spinal sensory neurons after nerve injury. J. Neurosci. 17, 3503-3514.

50. Sleeper, A.A., Cummins, T.R., Dib-Hajj, S.D., Hormuzdiar, W., Tyrrell, L., Waxman, S.G., and Black, J.A. (2000). Changes in expression of two tetrodotoxin-resistant sodium channels and their currents in dorsal root ganglion neurons after sciatic nerve injury but not rhizotomy. J. Neurosci. 20, 7279-7289.

51. Oyelese, A.A., Rizzo, M.A., Waxman, S.G., and Kocsis, J.D. (1997). Differential effects of NGF and BDNF on axotomy-induced changes in GABA(A)-receptor-mediated conductance and sodium currents in cutaneous afferent neurons. J. Neurophysiol. 78, 31-42.

52. Heyer, E.J., and Macdonald, R.L. (1982). Calcium- and sodiumdependent action potentials of mouse spinal cord and dorsal root ganglion neurons in cell culture. J. Neurophysiol. 47, 641-655.

53. Vergara, C., Latorre, R., Marrion, N.V., and Adelman, J.P. (1998). Calcium-activated potassium channels. Curr. Opin. Neurobiol. 8, 321329.

54. Margas, W., Ferron, L., Nieto-Rostro, M., Schwartz, A., and Dolphin, A.C. (2016). Effect of knockout of alpha2delta-1 on action potentials in mouse sensory neurons. Philos. Trans. R. Soc. Lond. B Biol. Sci. 371, 20150430.

55. Gao, L.L., McMullan, S., Djouhri, L., Acosta, C., Harper, A.A., and Lawson, S.N. (2012). Expression and properties of hyperpolarizationactivated current in rat dorsal root ganglion neurons with known sensory function. J. Physiol. 590, 4691-4705.

56. Weng, X., Smith, T., Sathish, J., and Djouhri, L. (2012). Chronic inflammatory pain is associated with increased excitability and hyperpolarization-activated current (Ih) in C- but not Adeltanociceptors. Pain 153, 900-914.

57. Passmore, G.M., Selyanko, A.A., Mistry, M., Al-Qatari, M., Marsh, S.J., Matthews, E.A., Dickenson, A.H., Brown, T.A., Burbidge, S.A., Main, M., and Brown, D.A. (2003). KCNQ/M currents in sensory neurons: significance for pain therapy. J. Neurosci. 23, 7227-7236.

58. Everill, B., and Kocsis, J.D. (1999). Reduction in potassium currents in identified cutaneous afferent dorsal root ganglion neurons after axotomy. J. Neurophysiol. 82, 700-708.

59. Sah, P., and Faber, E.S. (2002). Channels underlying neuronal calcium-activated potassium currents. Prog. Neurobiol. 66, 345-353.

60. Faber, E.S., and Sah, P. (2003). Calcium-activated potassium channels: multiple contributions to neuronal function. Neuroscientist 9 , 181-194.

61. Porreca, F., Lai, J., Bian, D., Wegert, S., Ossipov, M.H., Eglen, R.M., Kassotakis, L., Novakovic, S., Rabert, D.K., Sangameswaran, L., and Hunter, J.C. (1999). A comparison of the potential role of the tetrodotoxin-insensitive sodium channels, PN3/SNS and NaN/SNS2, in rat models of chronic pain. Proc. Natl. Acad. Sci. U. S. A. 96, 76407644.

62. Gold, M.S., Weinreich, D., Kim, C.S., Wang, R., Treanor, J., Porreca, F., and Lai, J. (2003). Redistribution of $\mathrm{Na}(\mathrm{V}) 1.8$ in uninjured axons enables neuropathic pain. J. Neurosci. 23, 158-166.

63. Boucher, T.J., and McMahon, S.B. (2001). Neurotrophic factors and neuropathic pain. Curr. Opin. Pharmacol. 1, 66-72.

64. Lai, J., Gold, M.S., Kim, C.S., Bian, D., Ossipov, M.H., Hunter, J.C., and Porreca, F. (2002). Inhibition of neuropathic pain by decreased expression of the tetrodotoxin-resistant sodium channel, NaV1.8. Pain 95, 143-152.

65. Zhang, W., Zhou, X., Wang, T.H., Wang, T.W., Liu, S., Chen, S.X., and Ou, K.Q. (2004). The neurotrophic effect of endogenous NT-3 from adult cat spared dorsal root ganglion on ganglionic neurons [in Chinese]. Sichuan Da Xue Xue Bao Yi Xue Ban 35, 25-28.

Address correspondence to: Laiche Djouhri, PhD

Department of Basic Medical Sciences College of Medicine (QU Health)

Qatar University

PO Box 2713

Doha

Qatar

E-mail: 1djouhri@qu.edu.qa 